\title{
Les séries de problèmes, un genre au carrefour des cultures : une première synthèse ${ }^{1}$
}

\author{
Alain Bernard ${ }^{\mathrm{a}}$ \\ Centre Koyré, 27 rue Damesme, 75013 Paris, Labex HASTEC ANR-10-LABX-85, France
}

\begin{abstract}
Résumé. Cet article propose une présentation du volume « les séries de problèmes, un genre au carrefour des cultures » et une vue d'ensemble des principales questions qui y sont abordées. La première partie décrit le contexte du projet qui y a donné naissance au sein du labex HASTEC, ainsi que l'objectif du volume et les conditions dans lesquelles il a été préparé. La seconde partie décrit le coeur du projet scientifique. On présente d'abord (2.1) une définition de son thème directeur, les problèmes qu'elle pose et les différentes manières dont il a été compris par les contributeurs ou contributrices. On résume ensuite (2.2) quatre grandes directions d'interprétation possibles des « séries de problèmes » en termes d'histoire culturelle : en lien à des visées encyclopédiques ; en lien à des fins pédagogiques ; comme objet de transformation et de réappropriation permanente ; comme support de légitimité savante.
\end{abstract}

\begin{abstract}
Series of problems, a genre at the crossroads of cultures: a first synthesis. This paper presents the volume entitled "series of problems, a genre at the crossroad of cultures" and offers a general view of the main questions that are addressed in it. The first part describes the institutional context of the project that gave rise to it within labex HASTEC, as well as the objective of this volume and the conditions in which it was prepared. The second part describes the core of the scientific project. It first presents (2.1) a definition of its leading subject, the problems this definition raises and the various ways in which it has been understood by the contributors. It then summarizes (2.2) four main directions of possible interpretation of "series of problems" in terms of cultural history: in relation to encyclopedic intentions; in relation to didactic ends; as an object of permanent transformation and re-appropriation; as support of scientific legitimacy.
\end{abstract}

\section{Préambule}

La présente introduction au volume collectif du même titre est divisée en deux grandes parties. La première explique le projet de recherche et de publication dans lequel elle s'inscrit, la manière dont ce collectif de chercheurs et d'étudiants s'est constitué, le mode de rédaction adopté pour le présent recueil

\footnotetext{
a e-mail : alain.bernard@u-pec.fr

${ }^{1}$ Ce texte introductif a été rédigé au cours de plusieurs réunions successives entre octobre 2014 et 2015, il s'agit donc en bonne partie d'une production collective (voir partie 1 et notes 4 à 6 plus bas). Plusieurs membres du groupe « séries de problèmes » ont en outre contribué aux dernières corrections, notamment Giovanna Cifoletti, Jean-Marie Coquard, Caroline Macé, Marc Moyon, Jeanne Peiffer, Bernard Vitrac. Qu'ils en soient ici remerciés.
}

This is an Open Access article distributed under the terms of the Creative Commons Attribution License 4.0, which permits unrestricted use, distribution, and reproduction in any medium, provided the original work is properly cited. 
et son rôle dans l'avenir du projet. Même si elle ne touche pas au cœur du projet et peut donc être sautée en première lecture, elle est néanmoins nécessaire pour comprendre l'esprit, l'objectif général et le mode de rédaction de ce volume.

La seconde partie présente un bilan d'étape du projet, sous la forme d'une version amplifiée et corrigée de son argumentaire : en partant d'une caractérisation du concept générique de " séries de problèmes » qui sert de point de départ, nous relevons et discutons les diverses interprétations qui en ont été faites au sein du groupe, ainsi que sur différentes interprétations en termes d'histoire culturelle qu'elle permet. Elle peut être lue soit comme une introduction aux contributions particulières, soit au contraire comme un complément qui indique les problématiques qui leur sont communes. La première étape de cette discussion (2.1) est probablement la plus ardue car elle aborde des questions fines de méthodologie. Les lecteurs pressés peuvent donc aller directement à la seconde partie (2.2), plus directement abordable car elle présente les interprétations historiques et culturelles proposées dans les différentes contributions. La première pose néanmoins des questions fondamentales qui ne devraient pas être ignorées, car elles sont des préalables nécessaires à toute interprétation.

Depuis son commencement, ce projet est associé à un carnet de recherche ${ }^{2}$ où sont détaillés certains aspects de nos réflexions. En particulier, il donne une idée des recherches de plusieurs participants ou participants au projet, qu'ils / elles aient contribué à ce volume ou non. Les lecteurs et lectrices sont donc convié-e-s, au delà des articles publiés dans ce volume, à explorer ce carnet qui présente quelques compléments utiles. Nous ferons nous-mêmes référence à certains billets dans cette introduction.

\section{Contexte et but de ce recueil}

\subsection{Le projet de recherche qui a conduit à ce volume}

Le projet de recherche interdisciplinaire "Les séries de problèmes, un genre au carrefour des cultures" est un projet pluriannuel qui a été initié au sein du laboratoire d'excellence HASTEC ${ }^{3}$ depuis sa fondation en 2011. Il a réuni pendant quatre ans sept collègues rattaché-e-s à quatre des laboratoires du Labex ${ }^{4}$, et une dizaine de collègues extérieur-e-s au Labex mais ayant participé activement aux travaux du groupe ${ }^{5}$, dont la moitié sont étudiant-e-s en master ou doctorat ${ }^{6}$. Cela représente donc en tout une vingtaine de personnes, sans compter des contributeurs-trices occasionnel-le-s ${ }^{7}$. Il ne s'agit aucunement d'un groupe clos et replié sur lui-même : il s'est en réalité constitué entre 2011 et 2014 par accrétions successives, à partir d'un trio de départ puis au fil des rencontres, les plus récentes remontant à 2015.

La diversité des champs disciplinaires couverts par ce collectif fait tout l'intérêt du projet : l'objet d'étude choisi nécessite en effet, comme on le verra plus loin, un tel croisement de perspectives. De ce point de vue, le sous-titre « un genre au carrefour des cultures » est à double entente. Il

\footnotetext{
2 http://problemata.hypotheses.org/ Par exemple, l'argumentaire initial du projet de recherche, auquel nous avons fait allusion, est lisible à l'adresse suivante http://problemata.hypotheses .org/28.

${ }^{3}$ Histoire et anthropologie des savoirs, des techniques et des croyances. ANR-10-LABX-85 http://www.hesam.eu/ labexhastec/ (consulté 6.10.14).

${ }^{4}$ Bernard Vitrac (CNRS) et Aurélien Berra (Université Paris-Ouest Nanterre) pour ANHIMA ; Giovanna Cifoletti (EHESS), Jeanne Peiffer (CNRS) et Alain Bernard (UPEC-ESPE) pour le Centre Alexandre Koyré ; Stéphane Lamassé (Université Paris I) pour le LAMOP ; Iolanda Ventura (Université d'Orléans) pour l'IRHT.

5 Pour les collègues en poste, il s'agit de Jeffrey Oaks (University of Indianapolis), Ursula Kundert (Université de Kiel et bibliothèque Herzog August, Wolfenbüttel), Caroline Macé (Goethe-Universität Frankfurt am Main), Marc Moyon (XLIM-DMI, Université de Limoges, membre associé au Centre A. Koyré), Jean Christianidis (Université d'Athènes, membre associé au Centre A. Koyré), Grégory Chambon (UBO, Centre François-Viète, membre associé au POCLAC).

${ }^{6}$ Il s'agit de Christelle Serra (enseignante en mathématiques, ex- étudiante en master EHESS), Fiancée Bantaba (doctorant EHESS) et Jean-Marie Coquard (ex-étudiant en master EHESS), tous élèves de G. Cifoletti en histoire des mathématiques ; Katalin Gosztonyi (doctorante en cotutelle entre le LDAR de l'Université Paris VII et l'Université de Szeged en Hongrie), Athanasia Megremi (doctorante à l'Université d'Athènes) enfin Francesca Aceto (doctorante au GAHOM - EHESS).

${ }^{7}$ Notamment Jean-Patrice Boudet (IRHT) et Sophie Démare-Lafont (Université Paris II et EPHE).
} 
désigne au premier chef les multiples éléments de contexte nécessaires à l'interprétation des séries de problèmes (nous y reviendrons plus bas, Sect. 2.2). Mais il renvoie aussi à ces champs de recherche multiples et à la rencontre entre des cultures intellectuelles et scientifiques différentes : histoire des textes, histoire du livre, anthropologie et histoire des pratiques intellectuelles, épistémologie et histoire des sciences, didactique des sciences, auxquelles il faut certainement ajouter le champ émergent des humanités numériques. La chose est suffisamment rare et originale pour être soulignée ici : il s'agit d'un authentique projet interdisciplinaire, pour lequel le Labex HASTEC offrait un cadre approprié.

\subsection{La nature intermédiaire de cette publication, le lectorat visé}

La nature ouverte et collaborative du projet permet de comprendre le caractère particulier de cette publication. Cette dernière ne doit pas être regardée comme un point terminal offrant des résultats totalement définis et élaborés. Il s'agit plutôt d'un premier point d'étape, qui nous conduira à une seconde phase du projet, où les questions soulevées ici pourront être approfondies en fonction de la lecture et du commentaire de textes choisis. L'objet principal n'est donc pas de lever toutes les ambiguiités ou les difficultés du projet, mais plutôt de faire un état des lieux des questions qu'il soulève pour l'instant, et des quelques réponses que nous pensons pouvoir offrir à ce stade. Autrement dit, nous ne sommes qu'au milieu du gué : il s'agit d'affiner les questions posées initialement en examinant divers dossiers et de montrer ainsi toute la mesure de notre objet d'étude, dans le cadre d'un projet de plus longue haleine.

Le lectorat attendu est au premier chef celui des chercheurs et chercheuses travaillant sur l'histoire et l'anthropologie des savoirs et des techniques, dans un sens large. Il intéressera notamment ceux d'entre eux qui étudient les phénomènes de sérialité dans d'autres sources historiques que celles qui reçoivent ici notre attention ${ }^{8}$. Ce lectorat inclut notamment les participants au Labex HASTEC qui connaissent le projet et ont lu les annonces ponctuelles de notre séminaire, mais que le contenu même de notre collaboration intrigue et qui aimeraient en savoir plus. Nous visons plus largement un public d'étudiante-s désireux-ses de s'initier à notre problématique de recherche, ainsi qu'un public de praticiens notamment les enseignant-e-s - pour lesquels la "mise en série" de questions de natures et de types divers reste une tradition vivante. Puisque nous avons rendu accessibles aux uns et aux autres certains des textes que nous étudions ${ }^{9}$, il était également naturel de leur rendre accessible un commentaire expliquant en profondeur l'intérêt que nous leur prêtons ${ }^{10}$.

\subsection{Le mode de rédaction du volume et de son introduction}

La première consigne adressée en 2014 aux futur-e-s contributeurs et contributrices était simple et adaptée à la nature du projet éditorial : chacun était invité à expliquer en quelques pages les raisons, spécifiquement liées à leur propre objet de recherche, qui les conduisaient à s'intéresser à la notion de « séries de problèmes » et à en faire soit un objet d'investigation, soit une notion heuristique. La seconde consigne était d'écrire de manière lisible et compréhensible pour un lectorat de non-spécialistes.

\footnotetext{
${ }^{8}$ C'est le cas, à titre d'exemple, des journées d'étude organisées au sein du Labex HASTEC autour de « l'histoire des listes », projet coordonné par Christine Lebeau, Gregorio Salinero, Roberto Bizzocchi et Arndt Brendecke. http://www. labex-hesam.eu/fr/hastec/49-pages/151-pour-faire-une-histoire-des-listes-letat-et-sessavoirs (consulté 28.11.14) ; ou encore du projet coordonné par Pierre Chastang et Laurent Feller sur « Listes et culture de l'écrit au Moyen Âge» au sein du LAMOP (http://lamop.univ-paris1.fr/spip.php?rubrique246, consulté 27.11.14).

${ }^{9}$ Depuis le départ en effet, le projet est associé à une formation d'un format original, s'adressant à un public mixte d'étudiants du Labex et d'enseignants en activité. Voir la rubrique "formation" de notre carnet de recherche.

${ }^{10}$ Nous évoquerons brièvement, dans la conclusion de la Sect. 2.2.3 ci-dessous, les études d'un genre différent, qui s'attachent à mettre en valeur l'intérêt de notre dossier documentaire pour l'enseignement ou la formation d'enseignants contemporains.
} 
Le mode de relecture des contributions a suivi un esprit semblable. Puisqu'il s'agissait de mutualiser nos réflexions personnelles et collectives en dépassant le cadre restreint de nos journées d'étude, nous avons opté pour (au moins) une double relecture interne au groupe : les participant-e-s ont d'une part été sollicité-e-s pour garantir la validité scientifique de chaque contribution (cohérence de l'argument, correction des erreurs factuelles et des lacunes), et d'autre part pour vérifier la lisibilité du texte pour un lectorat cultivé mais non spécialiste (clarté du propos, présence d'exemples accessibles, explicitations méthodologiques).

Tous les participant-e-s au projet n'ont pas forcément contribué à ce volume. Ce dernier ne reflète qu'une partie des contributions orales. Concernant la présente introduction, par contre, elle a été rédigée avec la collaboration d'une majorité de participant-e-s suivant le principe simple suivant : partir de l'argumentaire initial ${ }^{11}$ pour l'amplifier et l'affiner à la lumière des contributions disponibles - qu'elles aient été rédigées pour ce volume, mises en ligne sur le carnet de recherche ou présentées oralement durant les séances de séminaire. Sauf dans un cas ponctuel de co-rédaction (Sect. 2.2.2 ci-dessous) et compte tenu du fait que le texte a été relu et corrigé à plusieurs reprises ${ }^{12}$, la rédaction et l'architecture dans sa globalité me sont propres en grande partie, et j'en assume en particulier toutes les insuffisances ${ }^{13}$. Le but essentiel est d'aboutir à une vue d'ensemble du projet dans son état actuel, essentiellement au travers des questions et pistes de recherches qu'il soulève, et donc apte à intéresser le lecteur ou la lectrice à des problématiques qui ne sont pas forcément les siennes a priori.

Dans cette introduction, pour cette même raison, les références bibliographiques ont été volontairement réduites au minimum, c'est-à-dire pour l'essentiel à la liste des contributions de ce volume ainsi qu'à quelques publications des contributeurs et contributrices qu' on peut regarder comme complémentaires à ce volume. En effet, cette introduction n'a donc aucune prétention à faire un quelconque état de l'art sur une thématique qui dépasse de très loin les textes ou les corpus qui sont ici envisagés : une bibliographie exhaustive et une discussion de cette dernière serait bien trop vaste pour le cadre modeste de cette synthèse d'étape. En outre et surtout, la bibliographie pertinente pour chaque type de texte est indiquée dans les références des contributions particulières, et discutée plus en détail dans plusieurs d'entre elles, nous invitons donc les lecteurs à s'y reporter.

\section{Argumentaire scientifique du projet, études de cas}

\subsection{Un genre ambigu, à titre d'hypothèse}

\subsubsection{Au départ : un axe d'interprétation commun pour un corpus varié de sources}

Notre hypothèse de départ est qu'il y a possiblement un sens à comparer, sous l'angle de la question du genre, les méthodes d'étude de sources historiques aussi variées par le thème ou le contexte culturel que les suivants, que nous évoquons pour l'instant selon un ordre grosso modo chronologique : les multiples tablettes mésopotamiennes qui portent des énoncés sériels de problèmes mathématiques, médicaux, légaux, astrologiques ou comptables (G. Chambon) ; un corpus de textes anciens (en grec) ou médiévaux (en arabe ou en latin), transmis par des manuscrits et portant sur des problèmes à caractère métrologique (B. Vitrac, M. Moyon); les énigmes lettrées mêlant devinettes plaisantes et énigmes mathématiques de certaines sections de l'Anthologie Palatine (A. Berra); un ouvrage classique des mathématiques grecques anciennes, les Arithmétiques de Diophante (A. Bernard, J. Christianidis) ; les collections de questions-réponses en usage dans la littérature grecque chrétienne de l'Antiquité

\footnotetext{
11 Comme signalé plus haut, on peut consulter cet argumentaire de départ sur le carnet de recherche (http://problemata. hypotheses.org/28).

12 Voir note 1.

${ }^{13}$ Par souci d'homogénéité de la présentation, par ailleurs, j'évoquerai systématiquement ma propre contribution à ce volume, sur les Arithmétiques de Diophante d'Alexandrie, à la troisième personne.
} 
tardive au monde byzantin (C. Macé) ; la collection de problèmes constituant un traité d'algèbre en arabe au Moyen Âge (J. Oaks) ; la section arithmétique du quadrivium byzantin de G. Pachymère qui mêle explicitement les problèmes diophantiens et des techniques nicomachéennes (A. Megremi et J. Christianidis) ; les corpus foisonnants de textes encyclopédiques sous forme de questions-réponses portant sur des questions de philosophie naturelle en usage au Moyen Âge, à la Renaissance et jusqu'aux Temps Modernes (I. Ventura) ; le corpus non moins foisonnant des textes d'arithmétiques médiévales en latin ou en langue vernaculaire, étudié de longue date par les médiévistes (S. Lamassé) ; les textes mathématiques très variés et inventifs composés par des érudits renaissants comme Gemma Frisius (C. Serra), Simon Stevin (J.M. Coquard), Oronce Finé, Jean Borrel (G. Cifoletti), Luca Pacioli (F. Aceto) ; les recueils de disputationes publiés au 17è siècle en Allemagne (U. Kundert) ; les collections d'énigmes et de problèmes mathématiques publiés dans les journaux savants qui apparaissent aux 17è et 18è siècle (J. Peiffer) ; les manuels mathématiques de la réforme Varga dans la Hongrie des années 1970, défendant une approche de l'enseignement par problèmes (K. Gosztonyi).

Tous ces textes ont tout d'abord en commun d'être construits, au moins en partie, comme des séquences de questions et de réponses ou d'énoncés et de solutions ${ }^{14}$. Le second point commun est davantage d'ordre méthodologique, et revient à regarder tout ou partie de ces textes comme des « séries de problèmes », en désignant par là un genre de texte écrit, constitué d'un ensemble de questions et de réponses ou d'énoncés et de solutions, présentant un principe de cohérence. Dans ce projet nous considérons donc cette notion avant tout comme un objet de recherche interdisciplinaire et comme une catégorie méthodologique, que chacun a été conduit à spécifier en fonction de son objet d'étude, de façon à lui donner un caractère pertinent et opératoire.

On a vu en effet qu'une des raisons d'être de ce projet était de regrouper des chercheurs ainsi que des horizons, des méthodologies et des objets de recherche a priori différents (voir infra Sect. 1). Tous et toutes ces collègues ont cependant rejoint le projet parce qu'ils reconnaissaient dans son objet un axe d'interprétation intéressant pour leurs recherches, cette reconnaissance impliquant évidemment de spécifier la notion de série de problèmes en fonction de leur problématique de recherche. Le plus souvent, c'est précisément le degré d'accord ou de désaccord entre ces différentes manières de voir qui a permis d'approfondir la problématique collective et de mettre en regard différentes notions de séries de problèmes, soit pour les rapprocher, soit au contraire pour en percevoir les différences.

La définition large et floue de la notion générique de série de problèmes a donc fait, dans un premier temps, sa force et son attractivité. Elle a été le point de départ d'un questionnement collectif et la matière d'une réflexion pour chacun, puisque nous étions invités à déterminer ce que la catégorie nous permettait de voir dans nos sources.

Les commentaires qui suivent rendent donc compte de nos réflexions communes, aussi bien sur les éléments consensuels de cette caractérisation de départ que sur les façons éventuellement divergentes dont on pouvait la comprendre.

\subsubsection{Des questions et réponses, dans un sens général et englobant}

Dans la manière générale de décrire le premier point commun à nos objets d'étude, comme on l'a laissé entendre ci-dessus, les termes « question», «énoncé » d'un côté ou « réponse », « solution » de l'autre, prennent un sens très général. En effet, ils renvoient aussi bien aux questions de philosophie naturelle qu'on trouve dans les problêmata pseudo-aristotéliciens (ou dans leurs héritiers médiévaux), aux énigmes lettrées qui composent l'anthologie palatine ou le recueil foisonnant des « sophistes au

${ }^{14}$ La notion de «questions et réponses » est donc prise ici dans un sens bien plus large que ce que les spécialistes des littératures antique et médiévale comprennent sous le genre des « questions-réponses ». Nous reviendrons sur cette importante question dans la partie 1.4 infra, où nous renvoyons aux discussions plus spécialisées de C. Macé et I. Ventura dans ce volume. 
dîner » (ou «deipnosophistes ») d'Athénée, ou encore les problèmes métrologiques qui ont circulé de l'Antiquité au Moyen Âge.

Il y a une raison objective à vouloir ainsi regrouper toutes ces notions sous des termes communs qui permettent de les rapprocher, et cette raison est directement reliée au caractère singulièrement complexe de plusieurs des textes étudiés : certains d'entre eux, comme certains passages de l'Anthologie Palatine, des manuscrits médiévaux à l'exemple des Adevineaux amoureux ${ }^{15}$, des textes de la Renaissance humaniste comme les Opera Geometrica de Jean Borrel, les collections médiévales et renaissantes de questions réponses portant sur la philosophie naturelle, ou encore les collections disparates de questions qui circulaient dans les journaux savants au 18è siècle sous une rubrique dédiée, incorporent dans le même ensemble ces différentes sortes de questions. Ainsi, une énigme lettrée peut-elle voisiner, dans le même recueil, avec un problème arithmétique, une question de droit peut rencontrer un problème mathématique classique. Si dès le départ de nos rencontres, nous avons voulu nous confronter à des textes ou à des corpus qui présentaient une telle hétérogénéité disciplinaire apparente, c'est probablement parce que nous cherchions d'emblée à « tester » la validité d'une notion potentiellement englobante, sur des objets difficiles à catégoriser et pour lesquels elle pouvait s'avérer intéressante. Ce point crucial est mis particulièrement en évidence, dans ce volume, par les contributions de G. Cifoletti et I. Ventura au travers des exemples qu'elles étudient.

L'autre raison de regrouper ces notions sous un même terme générique est davantage de méthode. En effet les notions de problème, de question ou d'énigme, prises ensemble et d'une manière assez intuitive, renvoient en premier lieu à des réalités anthropologiques, notamment des activités humaines qu'on tient volontiers comme intemporelles, comme celle de poser des problèmes à autrui, de questionner à partir d'un certain matériau, ou de lancer des défis intellectuels ou culturels sur toutes sortes de questions. Dans les recherches d'anthropologie culturelle, c'est ainsi qu'on s'intéresse à de telles activités en les situant et les étudiant dans un contexte social précis. Pour des recherches historiques, cette réalité anthropologique reste une hypothèse théorique fondamentale. Elle permet de ne pas oublier que les sources écrites ne nous livrent qu'une petite partie de la réalité des mondes anciens ou récents : en particulier elles ne témoignent au mieux qu'indirectement de tout ce qui ressort à l'oralité, aux pratiques intellectuelles silencieuses et aux gestuelles codifiées.

Dans la mesure, donc, où les notions de problèmes, de questions ou d'énigmes sont polysémiques et sujettes à de fortes variations, y compris à l'intérieur d'un même espace culturel, il est utile de s'interroger sur le sens que nous leur prêtons à partir du moment où nous nous en servons pour interpréter des textes. À ce titre, le terme "problème" qui entre au titre du projet doit donc d'un côté être vu comme une question posée et un sujet de discussion pour l'interprétation des textes en termes d'histoire culturelle (cf. Sect. 2.2). D'un autre côté, il y a un consensus, bien reflété par les contributions, pour regarder le terme avant tout comme désignant des entités textuelles identifiables, comme nous allons le voir.

\subsubsection{Partir des textes et seulement d'eux : une précaution méthodologique}

Dans ce projet les notions de problème, de question ou d'énigme sont en effet d'abord comprises, dans un sens volontairement restrictif, comme les parties identifiables d'un texte écrit. Nos sources sont en effet avant tout écrites : même celles qui témoignent ou attestent une activité orale ou gestuelle ne le font qu'indirectement, par le biais d'un récit ou d'une collection d'indices matériels qu' on peut rapporter à des opérations pratiques. Les ensembles de questions et réponses ou d'énoncés et solutions qui nous

\footnotetext{
15 Voir l'annonce de la journée http: //problemata.hypotheses.org/114 (consulté le 15.10.15). Le contenu du manuscrit médiéval des Adevineaux Amoureux qui a été discuté lors de cette séance est consultable en ligne sur le site de l'université de Rennes. Ce document reproduit partiellement les Devinettes françaises du Moyen Âge éditées par Bruno Roy en 1977. La référence est donnée dans la bibliographie de l'article de S. Lamassé.
} 
intéressent posent des difficultés complexes de lecture et d'interprétation, ces dernières étant sujettes à de fortes variations suivant les hypothèses ou les méthodes d'interprétation retenues. Ils ne renvoient donc pas d'une manière univoque aux réalités anthropologiques que ces termes «problèmes », «questions », « énigmes » évoquent habituellement. Pour prendre un exemple bien connu, bon nombre de ces textes ont été interprétés comme des textes d'enseignement, c'est-à-dire lus comme des supports écrits dont l'usage serait analogue à celui que font nos écoliers de leurs livres d'exercices. Or, bien des traités anciens affichent par principe une intention didactique qui n'est qu' une codification culturelle courante et ne renvoie parfois à aucune réalité pratique ; ou bien, leur usage pédagogique n'est pas celui auquel nous pensons spontanément, en nous fondant sur nos propres apprentissages ${ }^{16}$.

Cette précision et cette prudence fondamentale vis-à-vis de nos sources textuelles se sont révélées particulièrement importantes pour les chercheurs et chercheuses de notre collectif qui contribuent à l'histoire des sciences et des mathématiques, ou encore à ceux qui s'appuient sur les matériaux des études ethnologiques et folkloriques. Dans de tels cadres, la notion de problème ou d'énigme est si courante, banale, éculée qu'elle en est souvent devenue un point aveugle, une fausse évidence. Que l'activité mathématique, par exemple, soit de tout temps une activité de résolution de problèmes est une idée qui fait facilement consensus et qui n'est bien sûr pas illégitime - d'autant plus qu'elle renvoie à une longue histoire. Elle possède toutefois l'inconvénient de brouiller la frontière entre une activité intellectuelle et ses manifestations écrites. En outre, même dans le cas où la distinction est maintenue, elle conduit trop facilement à privilégier la première, tenue pour la partie considérée la plus noble ou la plus digne d'attention, en occultant le détail des textes et de leur organisation.

Ces problèmes liés à la distinction entre textualité, oralité et gestuelle sont présents de manière plus subtile dans la notion même de texte. Ainsi pour les périodes antique et médiévale, les travaux d'anthropologie historique attentifs aux activités de lecture, de méditation ou de mémorisation conduisent à envisager sérieusement la possibilité qu'un ensemble sémantique cohérent puisse exister sans qu'il corresponde à une réalité écrite, mais plutôt à une réalité mentalisée, intériorisée par des procédés spécifiques «d'écriture spirituelle ». Dans cette perspective, il est légitime de penser que cette réalité mémorielle avait plus de valeur ou d'importance, aux yeux même des acteurs, que les textes écrits. Ce sont là des éléments d'interprétation qu'il faut absolument prendre en compte, y compris pour certaines des séries de problèmes qui nous intéressent. C'est donc sans naïveté, mais par méthode, que nous partons des documents écrits.

\subsubsection{Les séries de problèmes, une question de genre?}

Le sous-titre du projet «les séries de problèmes, un genre au carrefour des cultures » laisse volontairement entendre que la notion de séries de problèmes désigne un type, ou encore un genre littéraire. C'est ce que laisse encore entendre la caractérisation générale proposée ci-dessus (Sect. 2.1.1). Pourtant, en raison de la fonction essentiellement heuristique de la notion, ce « genre » est essentiellement fictif, il s'agit d'un pseudo-genre. Autrement dit, il ne s'agit pas pour nous en effet d'ajouter une catégorie éditoriale à celles existantes, d'autant plus que, comme on le verra plus loin, elle ne nous sert pas toujours à caractériser des textes dans leur intégralité (Sect. 2.5.1.3).

Si cette allusion à la théorie des genres littéraires s'est avérée cependant fructueuse pour notre projet, c'est qu'elle renvoie avant tout à une manière de questionner les textes bien connue des médiévistes, qui sont nombreux parmi les contributeurs et contributrices. Autrement dit, il s'agit bien davantage d'une allusion à une méthode opératoire d'investigation qu'à une catégorie nouvelle qu'il s'agirait de justifier.

En général en effet, le problème que doivent affronter les collègues qui étudient notamment un corpus large de textes est de différencier les textes non seulement par leurs contenus mais aussi par leurs genres et leurs sous-genres. Il s'agit, autrement dit, de catégoriser des types de documents écrits pour

16 Nous reviendrons plus bas (Sect. 2.2.3) sur ce type d'interprétation de nos textes relativement à un contexte d'enseignement ou à des visées didactiques. 
en comprendre la fonction, le lectorat, le statut ou encore pour étudier des effets de complémentarité au sein d'une même pratique érudite.

Dans le projet « séries de problèmes », comme on l'a vu, la notion de « collection de questions-réponses » doit s'entendre dans un sens beaucoup plus large, englobant et générique que ce que les historiens de la littérature antique ou médiévale entendent par là. Néanmoins, l'orientation méthodologique suivie présente un certain degré d'analogie à la leur : il s'agit de déterminer une catégorie opératoire qui permette soit de rapprocher des textes entre eux, soit au contraire de les distinguer. On trouvera dans le volume deux discussions très détaillées de ce type, restreintes à des collections de textes délimitées : un certain type de collections de questions-réponses ayant été élaborées en milieu grec chrétien entre le 5è et le 8è siècle pour C. Macé ; d'autres collections de questionsréponses d'époques renaissante et moderne et portant essentiellement sur des questions de philosophie naturelle pour I. Ventura ${ }^{17}$. À chaque fois un des intérêts de la discussion est de faire entendre les subtilités de la méthode employée pour distinguer un type de textes d'un autre, au sein d'un corpus large au sein duquel il faut faire des distinctions.

\subsubsection{Les problèmes et leurs sérialités possibles : quelques typologies envisageables}

Comme on l'a vu ci-dessus la notion de série de problèmes n'est pas univoque mais un objet de discussion, susceptible de compréhensions plus ou moins concordantes. nous avons retenu ici au moins trois typologies de ces différents niveaux de compréhension : en termes « d'intentionnalité sousentendue » plus ou moins forte (1.5.1), en termes historiographiques (1.5.2), enfin en termes de niveau d'appréhension des textes, sur un spectre allant du local au global (1.5.3).

\subsubsection{Sérialité intentionnelle ou fortuite?}

Le terme de série, en général, semble sous-entendre a priori un ordre intentionnel dans les éléments qui « font série » c'est ce qu' on pourrait appeler le sens « fort » des séries ou de la sérialité. Or donner un tel sens « intentionnel » à la sérialité paraît justifié dans le cas de séries de problèmes pour lesquelles nous avons des éléments d'interprétation, notamment des éléments d'introduction, de préface, ou de commentaire, qui permettent en effet de mettre à jour une intentionnalité plausible, rendant lisible un principe d'ordonnancement. Même quand ces déclarations explicites manquent, des éléments d'histoire culturelle, comme nous le verrons plus loin (Sect. 2.2), permettent dans certains cas de reconstituer cette intentionnalité. Dans le cas où un principe logique ou algorithmique semble gouverner une séquence, comme c'est souvent le cas avec des problèmes mathématiques, il est également tentant de le rapporter à une intentionnalité forte soutenant le raisonnement sous-jacent. Il en va de même, comme l'indique I. Ventura, pour certains groupes de questions-réponses touchant à la philosophie naturelle, qui peuvent être parfois regroupées suivant une structure intentionnelle ou du moins interprétable comme telle : c'est l'un des critères qu'elle retient pour catégoriser ces textes.

Dans d'autres cas cependant, le fait qu'un principe d'ordre intentionnel soit absent (au sens faible qu'il reste indécelable et qu'on ne puisse en faire la preuve) ne signifie pas pour autant que la série, ou collection, de problèmes n'ait pas une identité propre. Cette dernière n'est plus alors le produit d'un ordonnancement volontaire, mais provient du fait qu'une collection donnée a été reprise parce qu'elle était traditionnelle et/ou offrait des commodités pratiques pour bâtir de nouvelles séries sur le «fond culturel» des anciennes. Dans les discussions sur ce type de séries, il est possible, comme le fait S. Lamassé, de parler de «séquence textuelle» ou de «segments », donc en l'occurrence de

${ }^{17}$ A cette liste on peut ajouter l'étude des disputationes publiés en allemand au début du 17è siècle pour U. Kundert, qui a participé au séminaire du groupe en 2014. Voir http://problemata.hypotheses.org/331 (consulté 1.11.15) et le résumé attaché à ce billet. 
« séquences de problèmes » pour ce type de collection. J. Oaks préfère parler de « sérialité historique », mais avec un sens proche.

Le problème de cette différenciation est probablement que son association à la notion d'intentionnalité est discutable : il reste en effet difficile dans ce cas d'affirmer que cette conservation n'est pas elle-même le produit d'une intentionnalité indirecte, qui ne s'exerce plus dans le fait d'ordonner volontairement des éléments de questions réponses pour bâtir des séries nouvelles, mais bien dans le fait de reprendre des séries parce qu'elles ont - sans exclusive - un caractère d'ancienneté, de commodité ou un prestige qui leur donne un sens aux yeux des acteurs. C'est ce que S. Lamassé souligne, par exemple, lorsqu'il renvoie à la volonté des auteurs qui l'intéressent, de préserver une culture. En réalité, comme le souligne B. Vitrac, ce sont ici deux critères d'identification des séries qui entrent en jeu : un critère plutôt logique d'un côté, un critère relatif à l'histoire des textes de l'autre.

L'autre raison majeure pour rester prudent au sujet de la recherche des « intentionnalités cachées » derrière les séquences de problèmes dont on questionne l'ordonnancement, est qu'il existe encore des séries de problèmes dont le principe d'ordre semble très précisément être la recherche volontaire du désordre, par exemple à des fins «encyclopédiques » et/ou divertissantes. On verra (Sect. 2.2.2) que c'est le cas étudié dans plusieurs des contributions de ce volume où les séries sont regardées pour leur caractère ludique ou leur variété. Dans ce cas, qui intéresse tout particulièrement les séries ayant une forte valence culturelle et encyclopédique, on ne cherche pas un ordonnancement, ni non plus à conserver ou reprendre simplement ce qui existait par effet de tradition : il y a une intentionnalité, mais elle tient dans le fait d'englober et, en un sens, de désordonner.

En réfléchissant rétrospectivement à nos travaux, nous avons trouvé par ailleurs qu'il était utile de réfléchir sur la provenance historiographique des mises en série.

\subsubsection{Sérialité « autochtone », historiographique ou inventée ?}

D'un point de vue historiographique, les différentes contributions de cet ouvrage montrent que la notion de « séries de problèmes » est comprise à au moins trois niveaux différents, selon qu'on s'intéresse au sens conféré par les acteurs (sens « autochtone»), aux catégories historiographiques dont nous héritons ou enfin aux critères que nous sommes conduits à inventer.

Ainsi dans certains cas, l'objet de la recherche est principalement de repérer des catégories d'acteurs dans lesquelles on peut reconnaître un équivalent de notre notion. Pour prendre l'exemple éclairant proposé par U. Kundert au sujet des disputationes publiées au début du 17è siècle en allemand, certaines d'entre elles sont de pures parodies des disputes ordinaires propres au monde universitaire : la parodie montre simplement que même si le sujet est futile et très éloigné des sujets «normaux »de disputes, la forme «disputatio » de questions-réponses est suffisamment établie pour être reconnue. Réciproquement, ces pastiches représentent un observatoire privilégié pour comprendre la manière dont cette forme était comprise. U. Kundert nous a encore montré que l'examen de recueils de disputes-peut prendre la même fonction : le fait de réunir ces textes dans un même recueil peut devenir significatif de ce qui est le point commun entre eux ${ }^{18}$.

Dans d'autres cas, la recherche met l'accent sur l'élaboration explicite de nouveaux cadres analytiques qui permettent de mieux comprendre la structure d'un corpus de séries de problèmes. Ces cadres et ces catégories ad hoc, bien qu'ils ne soient pas arbitraires, n'en sont pas moins inventés pour les besoins de l'analyse : nous reviendrons sur ce point dans la section suivante (Sect. 1.1.6) qui est spécifiquement dédiée à cette importante question.

\footnotetext{
18 Mme Kundert n'a pu malheureusement contribuer à ce volume ; on pourra consulter néanmoins le résumé de son intervention de 2014, publié sur notre carnet de recherche (http://problemata.hypotheses.org/331) ainsi que le volume qu'elle a édité en 2010 avec Marion Gindhart : Disputatio (1200-1800). Form, Funktion und Wirkung eines Leitmediums universitärer Wissenskultur (de Gruyter 2010).
} 
Enfin, dans presque tous les cas, il n'est pas possible d'étudier les séries de problèmes qui nous intéressent sans prendre en compte l'état de l'art, c'est-à-dire les catégories ou les inventaires hérités de l'historiographie classique ou récente. C'est ce que montre en particulier l'étude de S. Lamassé qui s'appuie, jusque dans la définition du corpus qui lui sert de point de départ, sur une historiographie déjà très riche et incontournable, ou encore l'étude de B. Vitrac, qui part d'une déconstruction minutieuse de la manière dont on a artificiellement recomposé, au début du 20è siècle, le corpus métrologique qui fait l'objet de ses études. Ainsi, nos toutes premières discussions se sont portées sur la discussion des présupposés et des points aveugles d'un article célèbre de Warren Van Egmond, portant spécifiquement sur des problèmes arithmétiques mais posant des questions générales sur l'objet « problèmes » ${ }^{19}$. Van Egmond se proposait d'établir un inventaire, non des séries de problèmes, mais bien des problèmes eux-mêmes, sous une forme «hypostasiée » ou abstraite qui en rendait l'identité reconnaissable sur de très longues périodes de temps, comme l'expliquent M. Moyon ou B. Vitrac dans leurs contributions respectives. Nous dépendons évidemment de ces travaux et des riches perspectives qu'ils ont ouvertes, tout en étant obligé d'en critiquer certains aspects : en l'occurrence, le fait d'hypostasier les problèmes oblige par définition à faire passer au second plan les séries textuelles, c'est-à-dire certaines particularités structurelles de la documentation écrite d'où ils sont tirés.

Autrement dit, et selon le point de vue où l'on se place, la notion de série n'a pas la même fonction méthodologique ni la même valeur historique. D'un autre côté, il s'avère difficile voire impossible en pratique de distinguer ces trois types d'approche dans une même étude : en réalité les trois sont généralement présentes, avec simplement des dosages différents d'une étude à l'autre. Pour prendre l'exemple caractéristique de l'étude de B. Vitrac sur la partie « héronienne et pseudohéronienne » (donc grecque) des textes de problèmes métrologiques anciens, on y trouvera, comme on l'a vu, une critique des catégories historiographiques par lesquelles les textes de ce corpus ont été édités au 19è siècle ; mais aussi une discussion du sens donné à la notion de « séries de problèmes » dans le cadre de son étude ${ }^{20}$; enfin une discussion des catégories d'acteurs, en l'espèce des différentes attributions ou des titres des parties du corpus, qui indiquent entre autres le poids joué par la géométrie savante dans la manière de catégoriser et d'ordonner ces problèmes.

Mais cette lecture à plusieurs niveaux peut aussi se lire dans les termes d'une autre typologie, qui touche au caractère plus ou moins localisé de ce qu'on reconnaît sous le terme de « séries de problèmes ».

\subsubsection{Sérialité locale ou globale?}

Comme on l'a vu plus haut en effet, à partir du moment où l'on regarde la notion de série de problèmes comme se rapportant à une sorte de genre ou de pseudo-genre, on est également tenté de l'entendre comme désignant le tout d'une collection de questions et réponses. Or cette compréhension « globale » paraît contradictoire avec ce qu'énonce explicitement B. Vitrac dans sa contribution, et qu'expriment indirectement plusieurs contributeurs ou contributrices (M. Moyon, S. Lamassé, J. Oaks, K. Gosztonyi, A. Bernard) qui s'intéressent à des sous-parties très spécifiques de leur corpus : à savoir, qu'il faut comprendre par « série » un niveau intermédiaire d'analyse textuelle. Ce dernier se situe entre le problème « unité élémentaire », et la collection, qui est un texte ayant une identité globale identifiable par un certain nombre de caractéristiques : une attribution éventuelle, un titre, des marqueurs textuels spécifiques (traits de séparation par exemple). Dans ce cas il s'agit donc d'une unité d'analyse, qu'on ne peut pas dès lors confondre avec le texte entier dans laquelle elle s'insère et prend sens. Comprise ainsi dans un sens local, comme sous-unité identifiable d'une collection, les questions essentielles qui portent

\footnotetext{
${ }^{19}$ Pour une discussion historiographique plus étendue, voir les contributions de S. Lamassé, B. Vitrac ou encore M. Moyon. L'article de Van Egmond est discuté et référencé dans les trois.

20 À savoir, une catégorie opératoire pour étudier la structure interne d'un corpus, dont il montre au passage qu'il a une vraie cohérence. Nous revenons sur ce point dans la partie suivante (1.5.3).
} 
sur ce type de séries concernent la clarification des critères textuels et/ou logique qui les identifient (comme on l'a vu plus haut, Sect. 1.5.1) et bien sûr l'intérêt de repérer un tel niveau d'analyse.

Pourtant la notion de « série » est à l'évidence employée aussi comme désignant un tout, parce qu'en réalité on n'a pas alors en tête une séquence continue de problèmes qui présenterait une forme d'ordonnancement continu, mais plutôt un niveau de sérialité en quelque sorte structurant et supérieur. Comme le montre par exemple la contribution de C. Macé, c'est alors, d'une certaine manière, la mise en série elle-même qui peut être un marqueur de genre. D'autre part, la vitalité du processus de questionnement « réel », imité et revendiqué dans le genre littéraire, dépend du caractère « libre »de l'enchaînement des questions et des réponses. Ainsi, le principe de mise en ordre d'une série de questions et réponses opère plutôt par associations plus ou moins libres que par une construction intellectuelle définie.

Cette distinction s'avère d'autant plus intéressante à opérer que, dans plusieurs cas étudiés ici, les deux catégories de sérialité, locale et globale, doivent être considérées ensemble car les deux sont structurantes. Ainsi M. Moyon, dans sa contribution sur les textes métrologiques arabo-latins, distingue un premier niveau de sérialité qui renvoie à un ordre des figures élémentaires, généralement hérité de la géométrie savante, qui structure globalement ses textes, d'un second niveau, plus local et séquentiel, qui renvoie à une séquence continue répondant à des variations sur chaque figure. La figure de référence apparait ainsi comme un élément de structuration pour les deux niveaux. Non moins intéressante du point de vue de la « conjugaison » des deux niveaux de sérialité, est la contribution de J. Oaks : il montre par l'analyse le texte d'algèbre médiévale d'al-Sulamī, jusqu'ici peu étudié, que des critères d'ordonnancement locaux du traité antérieur d'al-Khwārizmī deviennent des critères d'organisation globaux chez al-Sulamī ; et qu'inversement que ce qui sert de « têtes de chapitre » chez al-Khwārizmī sert plutôt chez al-Sulamī à ordonner les séquences de problèmes qui composent chacun de ces chapitres. Dans la comparaison des deux textes on voit donc ici s'échanger, en quelque sorte, les deux niveaux d'analyse.

Il semble donc intéressant de distinguer ces différents niveaux de sérialité, étant entendu que la hiérarchie peut se compliquer dans le cas de compositions encore plus sophistiquées : c'est ce que montre l'analyse de S. Lamassé, dont la modélisation retient plusieurs critères de hiérarchisation des textes, ou encore les contributions s'intéressant aux textes renaissants, dont l'organisation souvent très riche et subtile doit se lire à plusieurs niveaux. C'est ce que l'on voit encore dans l'étude de I. Ventura, où la question de la catégorisation des textes doit être analysée à différents niveaux dont celui des séquences structurées de questions-réponses, mais pas seulement.

\subsection{6 Édition, inventaire et modélisation des séries de problèmes}

Revenons enfin à l'importante question des sérialités « artificielles » mises en évidence par les historiens -et notamment les contributeurs et contributrices à ce volume- lorsqu'ils cherchent à analyser leurs textes par des outils analytiques ad hoc.

Pour comprendre ce problème, il faut probablement repartir de deux raisons majeures d'élaborer de tels outils : pour l'inventaire de corpus lacunaires de séries de problèmes d'une part, et pour l'analyse de certains d'entre eux de l'autre. On a fait en effet allusion plus haut au fait que les contributions de cet ouvrage se partagent entre celles qui s'intéressent à l'étude d'un corpus entier, comme font S. Lamassé, B. Vitrac, U. Kundert ou I. Ventura, celles qui s'intéressent au contraire à des auteurs ou ouvrages particuliers, comme font A. Bernard, J.M. Coquard, C. Serra, A. Megremi et J. Christianidis ; d'autres enfin qui s'intéressent aux deux à la fois, c'est-à-dire à des textes particuliers mais étudiés comme paradigmatiques d'une tradition ou d'un ensemble plus large de textes, comme font G. C. Cifoletti, J. Oaks, M. Moyon, C. Macé et K. Gosztonyi.

Cette rapide répartition des contributions montre qu'il y a ici, par nature même de l'objet, deux types de textes qui se répondent : des œuvres particulières, et des collections de textes envisagées et définies 
dans leur ensemble. Cette précision implique immédiatement un problème simple : disposons-nous vraiment des ensembles en question? Nous en faisons-nous une idée correcte ? Si on peut penser que les textes métrologiques grecs qui intéressent B. Vitrac sont assez bien connus (le problème portant plutôt sur la manière dont on peut y reconnaître un corpus), d'autres collections sont notoirement lacunaires : c'est certainement le cas des recueils de problèmes mathématiques en langue arabe, dont une partie importante dort dans les archives ou les bibliothèques; des arithmétiques ou des encyclopédies médiévales, dont l'inventaire est lui aussi incomplet. Les contributions des médiévistes tels que M. Moyon, S. Lamassé, qui implique un questionnement méthodologique sur la manière d'organiser de tels inventaires. Ces problèmes de modélisation préalable à la définition d'un corpus font ici principalement l'objet de la contribution de S. Lamassé, en lien direct avec ses recherches en cours sur les arithmétiques médiévales en langue vernaculaire, ainsi que des réflexions récapitulatives de I. Ventura sur une catégorisation cohérente des textes médiévaux et renaissants de questions-réponses qu'elle étudie depuis des années. On trouvera également dans la contribution de C. Macé des indications détaillées sur les problèmes de catégorisation du corpus spécifique de questions-réponses élaboré dans les milieux chrétiens, sur lequel elle travaille.

Un problème connexe, touchant à la fois l'intégralité d'un corpus et les œuvres particulières, est celui des principes d'édition de séries de problèmes. B. Vitrac interroge indirectement ce problème en posant comme on l'a vu plus haut les termes d'une critique des éditions classique du corpus pseudohéronien sur lequel il travaille depuis des années : il indique que ces éditions n'ont pas su prendre en compte la réalité codicologique des manuscrits et, surtout, la nature des objets ${ }^{21}$. Son étude apporte des éléments qui pourraient constituer à terme un jalon important dans une possible édition des séries de problèmes métrologiques du corpus pseudo-héronien.

Enfin le problème des méthodes d'interprétation des séries de problèmes ne touche pas seulement les questions d'inventaire ou d'édition : il touche aussi (sans exclusive d'ailleurs) aux méthodes d'analyse des textes, et notamment des textes particuliers. C'est ce qu'indique la contribution d'A. Bernard au sujet de l'étude de la progressivité des Arithmétiques de Diophante, ou encore celle de J.M. Coquard sur la structure des problèmes de l'Arithmétique de Stevin, et la meilleure manière de la représenter. Ce problème n'est donc pas isolé et a une portée plus générale, puisqu'elle touche toute étude de problèmes particuliers, qu'il faut pouvoir mettre en perspective par leur appartenance à une série : étudier la série, rendre compte de ses caractéristiques propres ou de son ordonnancement, suppose en pratique de disposer d'un modèle au sens fort, c'est-à-dire un mode de schématisation évolutif. C'est ce que montre très bien, par exemple, l'étude de K. Gosztonyi sur le texte de Rózsa Péter qui est un des éléments de l'ensemble documentaire qu'elle analyse : la structuration en questions et problèmes du texte n'a pas un caractère d'évidence immédiat, puisque c'est un texte volontairement rédigé de manière continue et qu'il faut lire à plusieurs niveaux. Cette structuration doit donc être mise en évidence par un modèle d'analyse et d'interprétation qui permette de comparer sa structure à celle d'autres textes ultérieurs, et qui s'en inspirent.

\subsection{Un objet pour l'histoire culturelle et l'épistémologie historique}

On a vu plus haut que la caractérisation de départ que nous proposons pour les séries de problèmes, comme un genre de textes écrits, vise à préparer une base sûre pour leur interprétation en fonction d'éléments de contexte pertinent. Cette interprétation peut être construite en termes de valeurs culturelles pour les communautés qui élaborent ou font usage de ces objets, de pratiques orales et gestuelles, ou encore de pratiques érudites sur les textes eux-mêmes : lecture, écriture et réécriture, commentaires et recompositions. C'est en ce sens qu'on peut entendre le sens du sous-titre du projet, un genre

\footnotetext{
21 Voir à ce sujet son étude détaillée sur le sujet, en annexe à l'édition critique des Metrica de Héron (2014, avec F. Acerbi - on retrouvera la référence précise dans sa contribution).
} 
au croisement des cultures : les cultures en question sont moins les cultures au sens banal et « civilisationnel » du terme (ce sens convient mal, le plus souvent, pour ce genre transculturel), que les cultures de métier, d'enseignement, d'érudition ou plus généralement les cultures lettrées qui permettent de situer précisément l'usage, la fonction ou la valeur des séries de problèmes.

Nous proposons dans cette seconde partie de récapituler tout d'abord quel rôle joue, dans les différentes contributions, les hypothèses faites en terme d'histoire culturelle (Sect. 2.2.1) ; puis nous discutons quelques exemples de contributions où les textes sont interprétés en fonction de visées globales, comme la variété ou l'encyclopédisme (Sect. 2.2.2). Nous présentons ensuite les contributions qui abordent l'épineuse question du contexte pédagogique souvent présupposé, à tort ou à raison, derrière les séries de problèmes (Sect. 2.2.3) ; puis celles qui évoquent les problèmes de ré-appropriation de collections de problèmes (Sect. 2.2.4) et enfin celles qui s'intéressent au rôle des séries de problèmes dans la constitution d'une forme de légitimité savante (Sect. 2.2.5).

\subsubsection{Les rôles possibles de l'histoire culturelle dans une interprétation en termes de séries de problèmes}

Dans l'ensemble des contributions de ce volume, on peut grossièrement distinguer trois types de raisonnements principaux, qui donnent chacun une place plus ou moins importante à des hypothèses formulées en termes d'histoire culturelle. Certaines contributions, comme celles de B. Vitrac, de S. Lamassé ou de M. Moyon prennent comme objets essentiels les critères d'analyse ou de définition d'un corpus de textes, sans idée de poser une hypothèse forte sur le contexte culturel et anthropologique sous-jacent. Elles préparent donc au mieux à de telles lectures, mais ces dernières sont développées ailleurs ${ }^{22}$.

D'autres contributions suivent essentiellement le même schéma de raisonnement, qu'on pourrait qualifier de «préparatoire », mais posent explicitement - en introduction ou en conclusion - des hypothèses d'interprétation en termes d'histoire culturelle ou d'anthropologie : c'est le cas de l'étude de C. Macé sur les particularités de quelques collections de questions-réponses écrites en grec dans le monde chrétien des 5è-8è siècles, l'analyse découchant sur quelques hypothèses d'interprétation de la structuration propre à ces traités pour les milieux monastiques qui les ont produits ; de l'étude de J. Oaks, qui justifie l'hypothèse que la structuration particulière au traité d'al-Sulamī a des finalités pédagogiques ; et en partie de l'étude M. Moyon, dont la conclusion propose, au sujet de textes particuliers, quelques hypothèses sur le contexte de rédaction.

Les autres contributions donnent une place plus fondamentale à l'approche culturelle ou anthropologique en posant des hypothèses fortes sur le contexte de rédaction des textes étudiés. Ces dernières débouchent alors sur une lecture interprétative détaillée des textes, à la lumière de cette hypothèse ou venant au contraire corroborer cette dernière. C'est ainsi que la contribution de J. Christianidis et A. Megremi est introduite par une discussion détaillée sur le contexte intellectuel et culturel dans lequel évoluait G. Pachymère, qui débouche sur une lecture approfondie de la structure très particulière de la partie arithmétique de son Quadrivium. Les trois études portant sur différents traités mathématiques de la Renaissance (G. Cifoletti sur deux textes de Borrel, J.M. Coquard sur l'Arithmétique de Stévin, C. Serra sur la Méthode facile de Gemma Frisius), partent toutes d'une hypothèse forte sur le contexte intellectuel et culturel qui expliquerait alors le renouvellement rapide des productions écrites : l'essor de l'imprimerie d'une part, la réforme de la rhétorique et/ou de la dialectique de l'autre. C'est encore dans cet esprit qu'il faut situer la contribution de K. Gosztonyi, qui récapitule un certain nombre de thèses fortes éclairant le contexte très particulier qui, en Hongrie contemporaine

\footnotetext{
22 Par exemple, concernant une première histoire et des éléments de contexte pour le développement du corpus métrologique pseudo-héronien, on pourra consulter l'annexe III de l'édition des Métriques de Héron par B. Vitrac et F. Acerbi (référence dans la bibliographie de l'article de B. Vitrac).
} 
et dès l'entre-deux-guerres, a permis l'élaboration d'une approche philosophique et pédagogique des mathématiques par la «mise en problèmes » de ce savoir : cette hypothèse fondamentale oriente ensuite la contribution vers l'interprétation de textes particuliers, que l'on peut décrire en termes de séries de problèmes.

À mi-chemin entre les deux approches enfin, on trouve les travaux d'U. Kundert, de I. Ventura et d'A. Bernard, qui mettent en regard une étude détaillée de la structuration de certains textes ${ }^{23}$ et les hypothèses qu'on peut tenter de faire pour interpréter cette structuration. Dans le premier cas il s'agit de s'interroger sur le sens possiblement structurant que donnent les contemporains aux recueils de disputes ; dans le second si les éléments de structuration des textes peuvent s'interpréter en fonction du contexte épistémologique et culturel de production, dans le dernier il s'agit de se demander si la progressivité revendiquée des problèmes en question, en rapport avec la pratique rhétorique dans l'Antiquité, correspond bien à celle qu'on peut étudier dans les problèmes eux-mêmes.

\subsubsection{Les séries de problèmes comme expressions de visées encyclopédiques et comme recherche de variété ${ }^{24}$}

On a fait allusion plus haut au fait que plusieurs des contributions de ce volume ${ }^{25}$ s'intéressent à des modes de sériations de problèmes, qui valorisent paradoxalement ce qu'on pourrait appeler un « savant désordre », autrement dit une recherche de variété dont le principe n'est précisément pas de répondre à un unique principe d'ordre mais plutôt de représenter une culture, un savoir partagé ou en discussion. Autrement dit, ce principe réside dans une visée encyclopédique ou plus généralement «culturelle », au sens du partage de pratiques lettrées de référence. L'intérêt du croisement de ces contributions tient au fait que les savoirs de référence (philosophie naturelle dans un cas, mathématiques dans les autres) ne sont pas les mêmes, non plus que les perspectives adoptées : I. Ventura et G. C. Cifoletti s'intéressent à la comparaison de plusieurs ouvrages dont elles étudient les caractéristiques communes et les différences, C. Serra et J.-M. Coquard s'intéressent à des ouvrages particuliers mais dont l'étude se rattache aux catégories discutées dans les deux autres contributions.

I. Ventura, dans sa récapitulation critique de plusieurs de ses propres travaux sur les textes encyclopédiques médiévaux et renaissants qu'elle étudie à la fois sous le point de vue de l'histoire du texte et des sciences qu'ils véhiculent, propose une réflexion globale sur l'historiographie récente des différents genres de textes médiévaux et renaissants qu'on a pu qualifier d'encyclopédiques. Elle pointe les problèmes posés par le sens même donné à cet adjectif qui porte sur des genres de textes éventuellement distincts. Parmi eux on compte les recueils de questions-réponses concernant la philosophie naturelle, dont elle propose une catégorisation fine qui permettrait d'en saisir la diversité. La discussion des critères pertinents pour cela la conduit à mettre en évidence ce qu'elle appelle la structure des questions, qu' on pourrait encore appeler leur ordonnancement ou « mise en série » suivant différents principes intentionnels. Elle note que ces derniers, à leur tour, doivent être interprétés en fonction de la nature de la discussion épistémologique en jeu, ainsi que des conditions concrètes de lecture ou d'étude de ces questions : c'est donc à cet endroit précis que la question du contexte culturel et épistémologique joue un rôle central dans l'interprétation et la catégorisation des textes.

Cette discussion méthodologique générale, portant sur un large corpus de textes et un empan chronologique large, lui permet de pointer le fait que, parmi les principes intentionnels en question,

\footnotetext{
23 Les disputationes imprimées en allemand au début du 17è siècle pour la première, les recueils de questions-réponses sur la philosophie naturelle pour la seconde, une partie des problèmes des Arithmétiques de Diophante pour le troisième. Rappelons qu'U. Kundert n'a pu malheureusement contribuer à ce volume (voir supra, Sect. 2.1.5.2).

24 Cette partie a été rédigée en commun avec G. Cifoletti.

25 Il s'agit principalement I. Ventura, G. Cifoletti, C. Serra et J.M. Coquard. On peut ajouter à cette liste le travail d'A. Berra sur les pratiques lettrées anciennes reflétées par les énigmes de l'Anthologie Palatine ou du recueil d'Athénée Les Sophistes au dîner, qui posent mutatis mutandis des problèmes semblables.
} 
certains ne relèvent pas directement d'une volonté d'ordonner les questions suivant un point de vue ou un thème particulier, mais qu'ils conduisent au contraire à diversifier les questions pour en indiquer la variété. Or c'est précisément le point de vue qui est retenu par G. Cifoletti pour l'étude de plusieurs ouvrages imprimés de la Renaissance ayant un contenu mathématique, quoique répondant à des genres différents, et qu'on retrouve en partie dans les études de cas proposées par C. Serra et J. M. Coquard. ${ }^{26}$

La varietas est elle-même une notion ancienne, tirée des rhétoriques grecque et latine. Elle exprime l'opportunité d'inclure dans un discours une richesse d'éléments différents. Cette notion était entendue d'abord dans un sens linguistique ou stylistique, par le fait d'inclure des langues et des élocutions diverses, mais elle concernait aussi le contenu, par le fait de représenter plusieurs facettes d'une question.

Les textes envisagés dans deux des contributions en question sont, en ordre chronologique, l'ouvrage d'arithmétique (1540) de Gemma Frisius, celui d'Oronce Fine (1531), ceux de géométrie (1554) et d'algèbre (1559) de Jean Borrel. G. Cifoletti et C. Serra montrent que les séries de problèmes présentées dans ces ouvrages constituent des variétés, des collections où la diversité a un sens. Il y a dans ces cas une véritable recherche dans la diversité, avec des références parfois explicites à la notion rhétorique de varietas - chez Borrel notamment.

On retrouve dans l'étude de J. M. Coquard sur deux ouvrages publiés par Simon Stevin la même année (1585), son Arithmétique et sa Dialectique, l'idée d'ordonner un savoir exposé par problèmes, suivant un principe d'ordre tiré de catégories rhétoriques, plus précisément d'une entreprise de refondation de la dialectique ancienne dont Stevin partage le projet avec plusieurs auteurs renaissants. Si la façon d'ordonner les savoirs, qui se traduit concrètement par des schémas arborescents à valeur heuristique dans les textes de Stevin, répond a priori à d'autres principes dialectiques que celui de la variété, on y retrouve cependant l'idée cardinale d'un projet encyclopédique ambitieux et général répondant à la variété des ouvrages, activités et intérêts de Stevin lui-même.

\subsubsection{Mise en série des problèmes à des fins pédagogiques ou didactiques}

On a vu plus haut ${ }^{27}$ que l'interprétation directe de textes ayant la forme de collection de questions, d'énigmes ou d'énoncés avec des solutions comme des ouvrages d'enseignement, et de l'ordonnancement de ces énoncés comme relevant d'une visée directement didactique, n'est assez souvent qu'une fausse évidence. Cette interprétation fait trop vite l'économie d'un examen attentif de la documentation et des conditions et des raisons pour lesquelles elle a été élaborée, et propose de recourir à un contexte en quelque sorte stéréotypé à force d'avoir été invoqué, celui d'une activité d'enseignement. Le fait qu'il existe dans l'environnement contemporain, depuis le 19è siècle, une littérature spécifique aux pratiques d'enseignement et d'apprentissage, qu'il s'agisse de manuels ou de livres de maîtres, y est probablement pour beaucoup. Encore l'interprétation exacte de cette documentation moderne n'est pas simple pour autant, comme le montre dans ce volume la contribution de K. Gosztonyi.

D'un autre côté, plusieurs des auteurs identifiés et connus des textes étudiés dans ce volume, comme par exemple George Pachymère, Oronce Fine, Jean Borrel, Gemma Frisius, Simon Stévin, Rósza Péter étaient notoirement des enseignants, voire des enseignants réputés, et leur travail porte indiscutablement la marque de leur savoir faire pédagogique. Même pour ceux dont nous ignorons tout ou presque (comme Diophante, al-Sulamī, les auteurs connus ou inconnus des textes métrologiques évoqués par B. Vitrac ou M. Moyon, ou encore des arithmétiques dont discute S. Lamassé), on peut, au vu de leurs productions, spéculer sur l'idée qu'ils avaient des pratiques d'enseignement ou de formation professionnelle en partie reflétées par leur mode d'écriture. Mais cela ne fait pas de leurs productions écrites des manuels ni

\footnotetext{
${ }^{26}$ Ces trois contributions renvoient à un groupe de recherche qui s'est développé à partir des séminaires de G.C. Cifoletti sur les rapports entre algèbre et rhétorique dialectique au 16e siècle. Voir le site de l'EHESS pour le programme détaillé de ces séminaires.

27 Supra. Sect. 2.1.3.
} 
n'oriente de manière univoque l'interprétation de ces textes. En particulier plusieurs de ces personnages étaient aussi (voire, avant tout) des érudits, des savants, des techniciens, des lettrés ayant des desseins nettement plus complexes que didactiques ou pédagogiques au sens où nous l'entendons désormais.

Tout le problème, si on tient à interpréter les séries de problèmes en lien à des visées ou des contextes didactiques, est donc d'une part de clarifier ce que l'on entend par là, et d'autre part de se donner des critères aussi précis que possibles pour démontrer, ou du moins conjecturer de manière plausible, que telle ou telle documentation avait effectivement un lien avec une pratique ou une visée didactiques, et surtout lequel ${ }^{28}$. L'intérêt des contributions de ce volume est que chacune illustre la variété et la complexité des réponses qu'on peut donner à ces questions, suivant la nature des documents, leurs caractéristiques matérielles, et ce qu'on connaît (ou non) de leur environnement d'élaboration ou d'usage.

Sans prétendre à l'exhaustivité, proposons ici une rapide typologie de ces réponses. Bon nombre des approches méthodologiques employées dans ce volume, on l'a vu, consistent à tirer avantage de l'inclusion d'un texte dans un corpus ou dans un genre identifié, pour tirer des conclusions quant à la nature du texte en question. Cette comparaison aboutit bien, dans certain cas, à défendre comme plausible une finalité pédagogique, comme ce que propose de faire J. Oaks au sujet du texte d'algèbre d'al-Sulamī, sa structure indiquant une probable finalité pédagogique, en fonction de la comparaison avec celle des traités dont il s'inspire. C'est le même raisonnement auquel B. Vitrac fait rapidement allusion pour justifier comme plausible une finalité pédagogique au corpus métrologique qui l'intéresse : elle ressort de la comparaison avec le corpus latin des agrimensores romains qui n'a pas les mêmes finalités ${ }^{29}$. C'est encore par la comparaison entre plusieurs éditions du même texte de Gemma Frisius, en sus de ce que l'on sait de l'usage du texte comme manuel, que C. Serra peut conjecturer en conclusion un changement de type d'enseignement et de lectorat, pour un texte qui avait par ailleurs un rapport avéré et connu à l'enseignement des mathématiques de son temps.

Mais dans d'autre cas, la comparaison aboutit à la conclusion inverse : ainsi A. Megremi et J. Christianidis soulignent que le Quadrivium de Pachymère dont ils étudient une des parties, ne peut être assimilé au type de manuels latins qui ont servi de base à l'enseignement des arts libéraux dans l'occident latin. I. Ventura souligne de son côté, au sujet de la structure des textes de questions-réponses auxquels elle s'intéresse, qu'un usage pédagogique est plutôt l'hypothèse la moins probable pour les textes renaissants et modernes. Dans les deux cas c'est plutôt la visée encyclopédique et la discussion érudite entre pairs qui prime sur d'autres finalités plausibles.

D'autres contributions partent plutôt (ou également) d'un examen des projets didactiques exposés par les auteurs - dans le cas, bien entendu, où ces projets sont effectivement explicités, par exemple dans le cadre d'une introduction. C'est le cas par exemple, comme A. Bernard l'indique dans sa contribution, de Diophante énonçant un projet sophistiqué d'agencement de problèmes arithmétiques en des termes qui font allusion aux idéaux d'une éducation rhétorique. On peut encore évoquer celui de Jean Borrel liant explicitement l'ordonnancement de son traité en problèmes variés, à un type bien particulier de lectorat auquel convient un certain type d'éducation, comme l'indique G. Cifoletti ; ou encore de Stevin liant à son tour la structure très élaborée de son traité d'arithmétique à un public d'autodidactes, comme le montre J.M. Coquard. Mentionnons enfin le cas de R. Péter, rédigeant un texte de mathématiques pour un lectorat cultivé et lettré reflétant un idéal d'enseignement, en suivant l'analyse qu'en fait K. Gosztonyi. Tout ceci ne dit rien d'un quelconque usage des textes, mais beaucoup sur les idéaux directeurs des auteurs, qu'ils empruntent à leur tour à un environnement de valeurs et d'idéaux propres

\footnotetext{
${ }^{28}$ Ce problème général est envisagé à partir d'autres exemples que les textes évoqués dans ce volume, dans le recueil collectif Scientific Sources and Teaching Contexts Throughout History : Problems and Perspectives que j'ai co-édité en 2014 avec Christine Proust et dont on trouvera la référence dans la bibliographie de la contribution de S. Lamassé, qui a contribué au volume en question.

${ }^{29}$ Voir, dans sa contribution, la référence à l'étude complémentaire à son édition des Métriques de Héron d'Alexandrie, étude dans laquelle cet argument est développé.
} 
à leur temps, à leur milieu et/ou au lectorat qu'ils visent. Dans ce cas la visée didactique constitue principalement un idéal structurant qui renvoie à un horizon de valeurs philosophiques ou culturelles.

En définitive, seule la contribution de K. Gosztonyi, dans ce volume, vise pour des raisons qui renvoient clairement à la nature de ses recherches en partie didactiques, à cerner au mieux une pratique d'enseignement originale, fondée sur une mise en série de problèmes dans le cœur même d'une activité pédagogique à la fois pratiquée et qu'il a été question d'institutionnaliser en partie dans le cadre de la réforme qu'elle étudie. Pourtant, même dans ce cas, elle montre que cette pratique n'est pas bien compréhensible si on ne la rapporte pas à une conception des mathématiques et à une vision idéalisée de leur enseignement, qui est exprimée par des textes aux visées complexes comme celui de R. Péter.

$$
* * *
$$

Les contributions révèlent donc autant de perspectives variées d'interprétation critique des séries de problèmes en lien à des visées pédagogiques réelles, idéales ou inexistantes (car les visées sont autres). De ces approches il faut distinguer une autre perspective d'étude (voire d'usage) de ces textes auxquels deux contributrices à ce volume font rapidement allusion dans l'introduction de leurs articles : celui de l'intérêt de cette documentation pour l'enseignement contemporain et pour la formation des enseignants. C'est ainsi que C. Serra, qui est enseignante de mathématiques en poste dans un collège, évoque rapidement les raisons professionnelles qu'elle a trouvées à étudier G. Frisius et à s'imprégner de sa «philosophie arithmétique » si particulière, ou que K. Gosztonyi, sur le versant didactique de ses recherches, indique l'intérêt que peut prendre l'étude de son corpus pour l'enseignement et la formation d'aujourd'hui ${ }^{30}$. Plus généralement plusieurs contributeurs de ce volume, parce qu'ils sont ou ont été eux-mêmes enseignants ou formateurs ont développé une telle approche de leur texte, et nous renvoyons à leurs publications ${ }^{31}$.

\subsubsection{Les séries de problèmes, objet de commentaires et de réappropriation}

On l'a indiqué plus haut, l'étude historique des textes ici considérés dépend étroitement de l'histoire des problèmes, questions ou énigmes. Or la tradition historiographique, particulièrement prégnante dans le cas de l'histoire des mathématiques ou dans les études sur les traditions d'énigmes, indique que les problèmes ont une existence longue qui s'étend sur des siècles voire des millénaires, et qu'ils ont circulé au-delà des barrières des cultures ou des civilisations. On s'est ainsi efforcé de reconnaître chez Diophante ou Héron des problèmes déjà attestés par les tablettes mésopotamiennes, ou encore dans les recueils scolaires modernes des problèmes remontant au Moyen Âge ou à l'Antiquité. Dans le cas des séries de problèmes, la même remarque semble a priori s'imposer : le cas du recueil élaboré dans la Chine ancienne des Neuf Chapitres sur les procédures mathématiques, récemment édité et étudié par Karine Chemla et Guo Shuchun (2005) est à cet égard paradigmatique. Le classique chinois, dont l'importance pour la tradition scolaire médiévale en Chine peut être comparée à celle des Éléments d'Euclide en occident, est structuré comme une collection de problèmes dont la sophistication et l'ordonnancement sont mis en valeur par ses commentaires.

Or précisément cette transformation du texte au cours des siècles, ne serait-ce que par l'ajout d'un appareil de commentaires, ou dans les cas les plus extrêmes par le démembrement et remembrement

\footnotetext{
30 C'est un point qu'elle développe plus longuement dans un article publié en ligne. http: //dx. doi .org/10.1051/shsconf / 20141304003 (consulté 28.10.2015).

31 C'est notamment mon cas et celui de M. Moyon, qui travaillons tous deux pour la formation des enseignants, dans le cadre des ESPE et des IREM. Nous renvoyons les lecteurs intéressés à nos pages personnelles respectives pour des références bibliographiques détaillées. En général, les lecteurs qui sont enseignants de mathématiques (ou d'autres disciplines..) sont également invités à découvrir les riches ressources produites par la commission inter-IREM histoire et épistémologie (http://www.univ-irem.fr/spip.php?rubrique15, consulté 29.10.15) ainsi que sur le site expert de l'école normale supérieure CultureMATH (http://culturemath.ens.fr/histoire-des-math\%C3\%A9matiques-283 consulté 29.10.15).
} 
d'un texte traditionnel, indique d'emblée que l'argument de la «pérennité séculaire » (voire millénaire) des traditions de problèmes, s'applique difficilement sous une forme directe aux séries de problèmes. On peut déjà en discuter la validité dans le cas des problèmes eux-mêmes, qui n'ont été bien souvent vus comme pérennes, comme nous l'avons indiqué, qu'au prix d'une abstraction considérable par rapport aux réalités textuelles censées « rendre compte » de cette instanciation de "problèmes idéaux ». Dans le cas des séries de problèmes entières, a posteriori, le problème de leur transmission sur un temps long semble difficile à poser comme instanciation d'un «genre permanent » et lui-même hypostasié.

Une des surprises intéressantes de nos études, néanmoins, est que cette hypothèse d'une stabilité de certains types de séries de problèmes n'est pas à écarter d'emblée. En effet les études classiques d'histoire du texte tendent volontiers à classer les séries de problèmes comme textes d'enseignement et par conséquent instables, car ouverts à toutes sortes d'effet de modification et de recomposition, comme on peut s'y attendre dans une littérature ayant des finalités didactiques. Or l'étude de C. Macé montre par exemple que les collections de questions-réponses qui apparaissent dans le contexte de la littérature patristique écrite en grec, présentent un remarquable caractère de stabilité en tant que collection, puisque celle-ci est recopiée comme telle, même si certaines questions-réponses peuvent circuler dans différentes collections. Cette caractéristique est liée, comme on l'a vu plus haut, aux interprétations qu'on peut faire des « finalités didactiques » en question, dont l'interprétation n'est pas univoque et qui peuvent renvoyer à un mode d'organisation livresque (Sect. 2.2.3).

Dans d'autres cas on aurait pu s'attendre au contraire à des effets très forts d'héritages textuels indirects, via des traductions, par exemple dans le cas du corpus métrologique ancien qui a eu globalement (au niveau du type de problèmes de mesurage étudiés) un héritage médiéval très riche en langue arabe ou hébraique puis latine notamment. Or ce cas s'avère plutôt décevant, du moins dans l'état actuel de nos recherches : si on retrouve bien des problèmes ou des critères de classification typiques qui rapprochent effectivement ces corpus éloignés par la distance temporelle et par leur ancrage culturel, on ne retrouve pas forcément de séquences de problèmes identifiables comme ayant été transmises de l'Antiquité au Moyen Âge, par exemple (voir sur ce point les conclusions des contribution de B. Vitrac et M. Moyon).

Quelque part à mi-chemin entre ces cas extrêmes, l'article d'A. Megremi et de J. Christianidis touche à l'étude des héritages de séries de problèmes sur le long terme. IIs montrent en effet de manière saisissante comment un érudit Byzantin, George Pachymère, s'est réapproprié une partie des problèmes des Arithmétiques de Diophante, traité qui jouissait déjà du prestige d'un ouvrage classique, pour en détourner la logique propre et l'incorporer à un recueil largement structuré suivant une topique nicomachéenne. Il y a donc là une étude montrant une double logique à la fois de "patrimonialisation" d'un auteur classique, dont les problèmes servent de référence et de modèle, et de recomposition en fonction de buts nouveaux, en l'occurrence encyclopédiques. Ce qui est pointé là est probablement un caractère commun à un nombre important de séries de problèmes quand on les regarde sur le long terme, qui est pour ainsi dire la «vitalité du genre », selon l'expression suggestive de I. Ventura, c'est-à-dire leur capacité d'adaptation dans une forme relativement stable, favorisant un travail de réappropriation permanent. C'est le même phénomène qu'on voit à l'œuvre dans les corpus d'arithmétique et d'algèbre étudiés par S. Lamassé, J. Oaks.

Si donc, en l'état actuel de nos recherches, la question de la pérennité de certaines séries de problèmes sur le long terme ne doit pas être négligée, il paraît intéressant de redéfinir la question même de la «diachronicité » des séries de problèmes dans les termes d'une histoire de réappropriations successives, à la fois au niveau des conditions de possibilité et des modalités de ce processus, ce qui constitue par excellence un problème d'histoire culturelle.

\subsubsection{Les séries de problèmes, source de légitimé savante ou experte}

Concluons cette introduction en évoquant une dernière problématique d'histoire culturelle pertinente pour l'étude de nos séries de problèmes. Elle relève davantage de la sociologie historique et, pour partie, 
de l'histoire des sciences. Car si la structure, la forme ou l'intentionnalité propre à la composition ou à l'usage de certaines séries de problèmes sont éclairées par ce que nous savons de milieux particuliers, de leurs pratiques ou de leurs idéaux, la réciproque en quelque sorte s'avère intéressante à étudier dans bien des cas. Autrement dit, certaines séries de problèmes, notamment celles qu'on peut étudier à l'échelle de corpus entiers, ont manifestement été des facteurs d'identification pour des communautés lettrées, érudites ou scientifiques. Il suffit à cet égard de prendre l'exemple très explicite de Jean Borrel : G. Cifoletti montre bien dans son étude que les réflexions portant sur les séries de problèmes qui intéressent Borrel, du point de vue de leur contenu comme de leur mode de composition, ne sont pas dissociables du type de communauté et du statut social visé. En l'occurrence, les séries de problèmes étudiées (c'est-à-dire celles que doivent étudier) les «logisticiens » ne sont pas les mêmes que celles des « calculateurs $»^{32}$.

Or ce problème est général et ne concerne pas seulement la question de ce que Bruno Belhoste et J. Peiffer ont d'abord théorisé, dans le cadre des premiers travaux du Labex, sous le terme de « mise en science $»^{33}$. Certes, dans bien des cas on peut soupçonner (voire prouver) que certains genres de séries de problèmes ont servi de référence pour la formation et la légitimation de communautés de spécialistes, praticiens, artisans ou savants puis «scientifiques » pour une période plus tardive. C'est ce qu'étudient J. Peiffer et ses collègues dans le cas des questions-réponses présentes dans les journaux savants du 17è au 18è siècles : cette «forme » des questions-réponses permettait de faire participer les lecteurs à l'élaboration des journaux et donc de créer des communautés de lecteurs et d'auteurs ${ }^{34}$. Mais il n'en va pas autrement pour les collections «variées », à vocation culturelle, qu'étudient A. Berra, F. Aceto ou, à nouveau, G.C. Cifoletti dans le cas des juristes renaissants. Il faut rappeler que la culture lettrée n'était pas, dans l'Antiquité ou au Moyen Âge, ce qu'elle est devenue pour nous, un objet de démocratisation et -au moins de manière souhaitable dans le cadre d'un système d'éducation obligatoire- un bien commun. Elle était plutôt l'apanage d'une élite qui défend ses privilèges et ses signes de reconnaissance au travers du partage actif de pratiques et de références, qui sont en partie véhiculées par des séries de problèmes. Même dans le cas des questions-réponses publiées au 18è siècle et qui faisaient l'objet d'une spécialisation croissante, l'appartenance à une culture lettrée (maîtrise du grec et du latin, etc.) pouvait se manifester au travers du mélange de séries d'énigmes (en langues anciennes) et de problèmes mathématiques.

Il y a donc là un nouvel horizon de questionnement, qui touche à la fois à la question de la constitution de milieux et tout à la fois de domaines spécialisés («mise en science »), et à celui du maintien de cultures érudites ou lettrées. À cet égard, tout l'intérêt du projet est de permettre de penser les premiers sur le fond des secondes. De ce point de vue, l'étude de séries de problèmes « mixtes », mélangeant énigmes lettrées et problèmes de sciences, prend une importance stratégique très nette.

\section{Références}

Bernard, Alain. 2015. Interpréter la sériation des problèmes des Arithmétiques de Diophante : une forme de modélisation? Dans ce volume 00003. EDP Sciences : SHS web of conferences.

Christianidis, Jean et Megremi, Athanasia. 2015. Theory of ratios in Nicomachus' Arithmetica and series of arithmetical problems in Pachymeres' Quadrivium : reflections about a possible relationship. Dans ce volume 00006. EDP Sciences : SHS web of conferences.

\footnotetext{
32 Cette réflexion, sous la plume d'un érudit de la Renaissance, n'est bien sûr pas seulement théorique ou programmatique : elle correspond à la réalité des programmes d'enseignement mis en place dans les collèges, où encore à celles des modes de publication ou de diffusion des textes érudits. Cette réalité est reflétée par le choix de la langue ou de la structure des traités, comme le montre notamment C. Serra dans le cas de Frisius.

33 Voir le texte de problématisation initial : http://www.labex-hesam.eu/images/labex/Hastec/Documents/Progrcollaboratifs/programme\%20collaboratif\%206.pdf (consulté le 19.10.2015).

${ }^{34}$ Pour plus de détails voir le billet de J. Peiffer http://problemata.hypotheses.org/389 (consulté 1.11.15).
} 


\section{SHS Web of Conferences}

Cifoletti, Giovanna. Renaissance: series of problems as varietas. Dans ce volume 00011. EDP Sciences : SHS web of conferences.

Coquard, Jean-Marie. 2015. Mathématiques et dialectique dans l'œuvre de Simon Stevin. L'intérêt des séries de problèmes. Dans ce volume 00012. EDP Sciences : SHS web of conferences.

Gosztonyi, Katalin. 2015. Séries de problèmes dans une tradition d'enseignement des mathématiques en Hongrie au 20e siècle. Dans ce volume 00013. EDP Sciences : SHS web of conferences.

Lamassé, Stéphane. 2015. Mise en série et tradition de problèmes dans les arithmétiques en langue vulgaire de la fin du Moyen Âge. Dans ce volume 00009. EDP Sciences : SHS web of conferences.

Macé, Caroline. 2015. Pseudo-Césaire, Anastase, Pseudo-Athanase : Quelques réflexions sur les collections de Questions-et-Réponses dans la littérature grecque chrétienne. Dans ce volume 00004. EDP Sciences : SHS web of conferences.

Moyon, Marc. 2015. Comprendre les géométries de la mesure par les « séries de problèmes ». L'exemple des pays d'Islam et de l'Occident latin du IXe au XIVe s. Dans ce volume 00007. EDP Sciences : SHS web of conferences.

Oaks, Jeffrey. 2015. Series of problems in Arabic algebra: The example of 'Alī al-Sulamī. Dans ce volume 00005. EDP Sciences : SHS web of conferences.

Serra, Christelle. 2015. Le rôle des problèmes dans la « Méthode facile d'arithmétique pratique » de Gemma Frisius. Dans ce volume 00010. EDP Sciences : SHS web of conferences.

Ventura, Iolanda. 2015. Les recueils de questions-réponses à l'Époque Moderne : une forme de transmission du savoir scientifique? Dans ce volume 00008. EDP Sciences : SHS web of conferences.

Vitrac, Bernard. 2015. Collections et séries de problèmes métrologiques dans le corpus héronien et pseudo-héronien. Dans ce volume 00002. EDP Sciences : SHS web of conferences. 